\title{
HCI for Real world Applications
}

\author{
Sreeji C, Vineetha G R, Amina Beevi A, Nasseena N, Neethu S S \\ Depariment of Computer Science and Engineering, Kerala University \\ Sree Buddha College of Engineering, Pattoor, Alappuzha
}

\begin{abstract}
Human-computer interaction (HCI) is necessary for different real world applications. Swarm robotics is an emerging technology in the real world. This paper presents navigation of swarm robot through hand gestures. The interaction of Human-robot is needed for controlling the swarm robots. The communication between multiple robots possible through sending messages. For sending messages Bluetooth technology can be used. Our goal is to make swarm robot to work effectively based on the gesture given. Background noise and dynamic environments are the key issues in the gesture recognition process. The background objects will affect the system performance and accuracy. Hand tracking along with feature extraction is used in this paper to dealing these issues. The robot used is the foot-bot robot developed in swarmanoid projects. Webcam is used as visual interface. Collaboration of robot with human has great importance in the field of service robotics.
\end{abstract}

Keywords - Swarm robots, hand detection, Gabor filter, gesture identification, navigation of swarm robots, Bluetooth, human-computer interaction.

\section{INTRODUCTION}

Nowadays, robots are used in various environments for different applications, from industrial fields, manufacturing to autonomous exploration of remote planets. Among these, mobile robots have achieved great consideration from scientific, industrial and military communities. Robotics deals with design, construction, manufacture, application of robots. These are automated machines that can work like humans. They can work in dangerous places, where humans cannot. The main characteristics of robots are sensing, movement, energy and intelligence.

Swarm robotics is an approach for coordinating a large number of relatively small robots. Swarm robotics is a field focuses on controlling large scale homogenous multi robot systems. Swarm robots have the characteristics like robustness, flexibility, scalability, effectiveness, fault tolerance, adaptability and so on. Robots used are simple and cheap. This paper presents how to co-ordinate a number of robots for performing a particular task. A powerful processor is required for control of swarm robots. The controller has additional task compared to individual mobile robot controllers. In addition to obstacle avoidance and navigation swarm robot can communicate through sending messages [4]. Swarm can perform tasks that one expensive robot cannot. Even if some of the robots in the swarm fail, it can still achieve the task. For swarm applications real robot is not used because of its high economical cost and large area needed for execution.

In this paper swarm robots move according to the commands given through gestures. We use hand gestures for giving commands. Which is the powerful way of communication and additional devices are not needed. A hand gesture is a movement that we make with our hand to give control instead of speaking. Recognizing gestures is a complex task which involves motion modelling, motion analysis, pattern recognition etc. Vision based interface are one of the key research area in which human-robot interaction gain interests [2]. In this paper we use tracking algorithm to detect hand in the image and Gabor filter for feature extraction and identification. Presence of noise is a serious issue. Unwanted objects and backgrounds will affect the system. So we use a tracking algorithm to detect hand and extract information from that part. We select skin color as a parameter for tracking a hand because of its computational simplicity. Hand gestures are very rich in shape variation, motion and textures. So we choose static features like finger tips, finger direction and hand contours are selected for recognition. But lightning conditions will not give correct features.

The rest of this paper is organized as follows. Section II gives a brief idea about location sensing systems and mobile robot navigation. Section III presents hand detection. Section IV presents feature extraction and gesture recognition. Section V presents goal directed navigation of swarm robots. Section VI presents hardware implementation and section VII includes conclusion.

\section{A. Location Sensing Systems [7]}

\section{Related Work}

Location sensing systems in mobile robot can be classified into absolute and relative sensors. Absolute location sensor includes GPS (global positing system) using at least three satellites and an external camera. Robots with GPS [11] receivers can obtain their own 3-D location plus time information. The receiver is smaller in size. GPS is difficult to deal with signal blockage, so will not work in indoors. 
The relative sensors include cameras, RSSI (received signal strength indicator) measurement systems and proximity sensors. Camera placed on robot take the snapshot of surroundings. Through a series of computations the information is extracted from the snapshot. From the continuous process, the robot can recognize the presence of objects.

RSSI measurement systems are employed in wireless environment based on wireless networking of IEEE 802.11 protocol family or RFID (radio frequency identification) RSSI measurement systems are not affected by any obstacles. RSSI needs other hardware devices like antenna and reader. Because of low accuracy, it is difficult to deploy large-scale robot swarms using RSSI measurements system.

Proximity sensors are classified into LRF (laser range finder), ultrasonic sensor and Ir (infrared sensor). Compared with LRF, sonar and Ir are smaller and low cost. These merits can be directly connected with swarm organization of larger number of mobile robots. Ir based parallax distance measurement can be cheaper and smaller than sonar, but it is affected by color of objects. Multi sensor fusion technique like camera plus laser photo detector and RSSI based on sonar introduced to make mutual strengths.

\section{B. Mobile Robot Navigation [13]}

For navigation of mobile robots in the indoor environment needs some tasks. Assume the environment to be static and all other objects are rigid. Indoor environment has intrinsic uncertainty and complexity that cannot be overcome with this approach. An alternative, reactive approach is used. An example is the use of potential field method (PFM) for local planning like obstacle avoidance. In the combination of global path planning with local path planning, calculate the shortest path between two points, as it navigates adjust the path plan according to the environment. Obstacle avoidance results naturally from the incremental path planning.

\section{HAND DETECTION}

This section deals with detection of hands by taking parameter as skin.

\section{A. Detecting Skin And Non-skin}

Color is used as a parameter in detection. Detection of hand from a dynamic environment is done by means of identifying color. So selection of color space is crucial. Geometric variations and orientations are the issues which are to be handled while tracking. The aim is to differentiate between skin and non-skin pixels.

Detection of skin includes detecting image pixels and regions that contain skin-tone color. Background should be controlled or make uneven to skin colored objects. The appearance of skin in an image depends on the illumination conditions. Skin detection takes place in two phases. Training phase and detection phase.

Training a skin detector involves three basic steps. Collect database of skin patches from different images. Choose an appropriate color space. Learn the parameters of skin classifier. Detection phase involves two steps. Converting the image into some color space and classifying each pixel using skin classifier to either skin or non-skin. Classifier classifies and labels whether it is a skin or non-skin. In the context of skin classification true positives are skin pixels and classifier labels it as skin and true negatives are non-skin pixels and label it non-skin. Most commonly used color space is RGB color space because of its simplicity. A variety of classification techniques can be used. Any pixel falls inside the skin color class boundary is labelled as skin.

\section{B. Detection Based On Skin}

After detecting the skin a threshold was defined to separate skin and non-skin. A binary image was generated which tracks the skin region and non-skin region. Intensity weighted centroid method is used to keep track of skin regions. Then the image frame is cropped so that the resultant image contains only hand region.

\section{FEATURE EXTRACTION AND GESTURE RECOGNITION}

\section{A. Gabor Filter}

Each of the images is passed through a bank of Gabor filters in 8 equally spaced directions and $\theta$ at 16 frequencies $\lambda$ to get their dense filter responses below

$$
G(X, Y)=\exp \left[\begin{array}{c}
-X^{2}+\gamma^{2} Y^{2} \\
2 \sigma^{2}
\end{array}\right] * \cos \left[\begin{array}{l}
\frac{2 \Pi}{\lambda} X \\
\end{array}\right]
$$

Where $X=x \cos \theta+y \sin \theta$ and $Y=-x \sin \theta+y \cos \theta$ are the rotations of the Gabor filters with angle $\theta$ which varies between 0 and п. Let each image by tessellated by squares at 8 different scales, where each scale corresponds to two adjacent Gabor filter banks of a compatible frequency. For each scale, the maximum-operation is performed on the two Gabor filter responses whose frequencies are nearest to the scale, on a pixel-wise basis. This scheme allows some robustness to slight mis-alignment during the pre-processing stage. Finally, at each scale, the 
variance is computed from the resultant responses within each square. These variances are finally concatenated to form a feature vector and features are stored in the database.

\section{B. Gesture Recognition}

Gesture recognition enables humans to interface with the machine and interact naturally without any mechanical devices. First hand is tracked and then features are extracted using Gabor filter from the image. Gabor filter operation is performed on two images, one is the model image (image from the database) and other is the test image. The database image contains a gesture which is taken in predefined conditions and captured in usual environment. Many matching techniques used are Manhattan distance, Euclidean distance etc for finding the match [12]. For a feature $\mathrm{F}_{1}^{\mathrm{i}}$ in a model image the corresponding feature $\mathrm{F}_{2}^{\mathrm{j}}$ must be checked in test image. If a pair of features matched Euclidean dist $\left(\mathrm{F}_{1}^{\mathrm{i}}, \mathrm{F}_{2}\right)$ should be calculated. A threshold is kept to determine whether match is positive or negative.

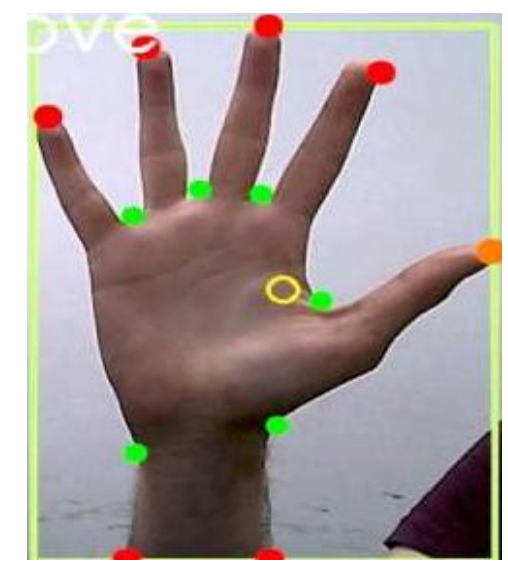

Fig. 1 Feature extraction

\section{GOAL DIRECTED NAVIGATION OF SWARM ROBOTS}

The task of the robots is to detect and understand the command, and collectively reach a distributed consensus about it in order to actuate its execution. The problem is particularly challenging since the robots in the swarm can be spread in different positions in the environment and be engaged in tasks of their own when the command is issued. We use hand gestures as mean for human-swarm communication. A hand gesture encodes a command that the swarm will execute. Hand gesture is a powerful way for communication. So no need of additional devices.

We investigated how to exploit robot mobility, swarm spatial distribution, and bluetooth communications, to let the robots in the swarm: (i) implement a distributed and cooperative sensing of hand gestures, and (ii) robustly reach a consensus about a gesture. Motorized track-based wheels allow a robot to move at a specified speed. System gets input from the webcam and gesture recognition is performed. The message propagates between swarm by using bluetooth communication system.

\section{HARDWARE IMPLEMENTATION}

Real time implementation of hand gesture recognition consists of foot-bot robot developed in swarmanoid project. The foot-bot robot developed mainly for swarm applications. We use a subset of sensors and actuators available for such platform. The frontal camera is used for recognizing gestures. Motorized track based wheels allow robot to move at the speed of $5 \mathrm{~cm}$ per second. The infrared-based-range and bearing sensor and actuator allow a robot to detect its line of sight neighbours up to a range of few meters and to recover their distance and bearing. The system gets input from webcam. After acquiring gestures, we use Gabor filter feature extraction individual gesture recognition and generation of opinion vectors, assigning probability to each known vectors. The resulting opinions are spread to all robots in the swarm through sending messages. Each robot can record its own opinions and opinions from other robots. Generate a decision vector as the component sum of all classification vectors D or opinions it was locally generated and/ or received from other robots. D's component with the highest value, $i^{\prime}$, indicates the gesture class in favour of which most evidence is available at the moment to the robot. The robot also calculates its measure of confidence about the true class being $\mathrm{i}^{\prime}$ as $\lambda=\mathrm{Di}^{\prime}-$ $\mathrm{Di}^{\prime \prime}$. Where $\mathrm{i}^{\prime \prime}$ is the index of the second highest component of $\mathrm{D}$.

When a robot has gathered enough evidence, $\lambda$ exceed a predefined threshold, send it to swarm. It propagates through bluetooth communication. Robots receiving a decision immediately adopt it. If different 
decisions are generated in the swarm the one accessed with the highest confidence overrides the propagation of others.

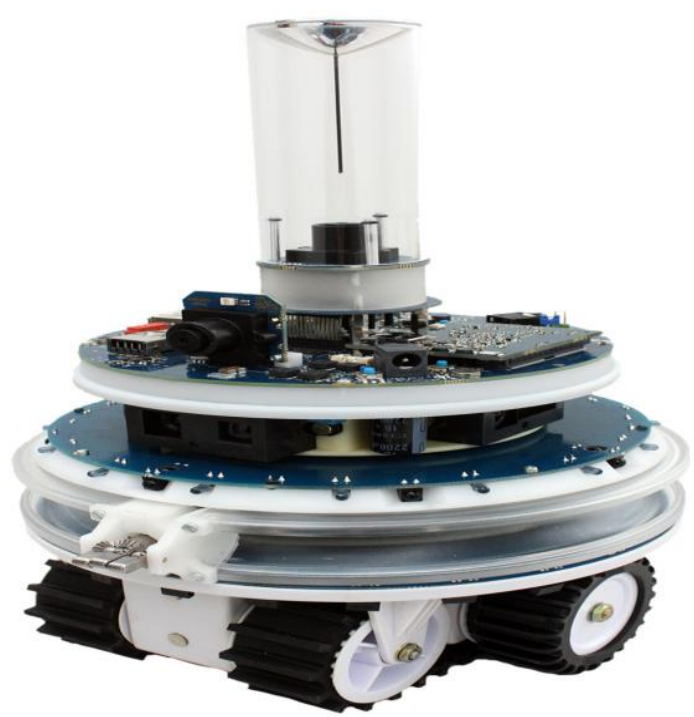

Fig. 2 Hardware implementation of hand gesture recognition system.

Robots start acquiring hand images at a rate of roughly one per second. Immediately after each acquisition, the image is processed as described above. Resulting decisions are spread to robots in the swarm.

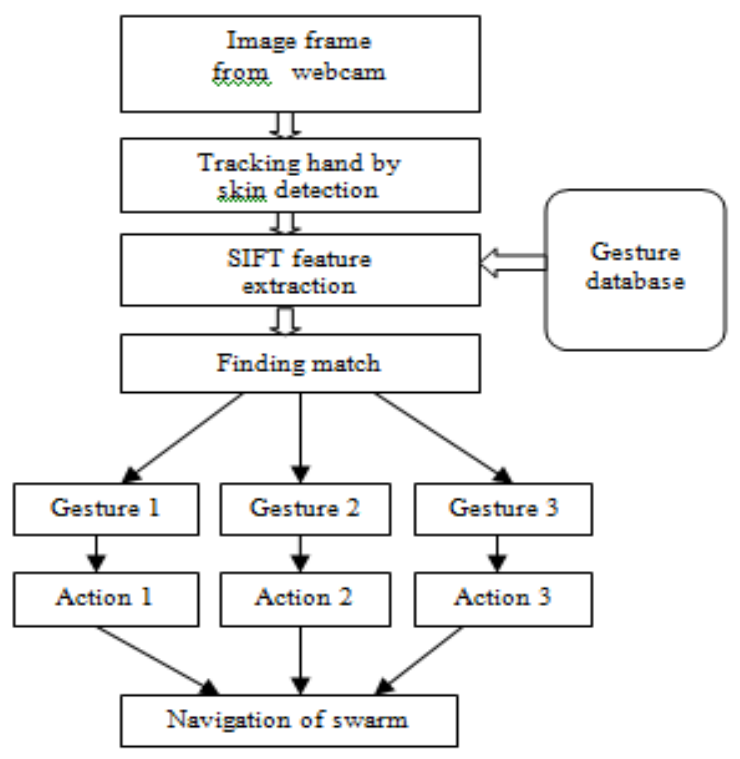

Fig. 3 Hand gesture recognition and navigation system

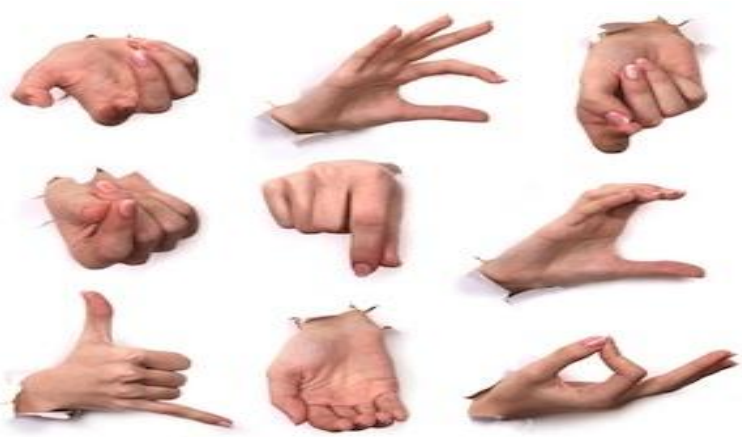

Fig. 4 Different hand gestures used to create database 


\section{CONCLUSION}

In this paper we provide the facility of gesture recognition to mobile robots for swarm applications. The communication between robots is possible by sending messages. Features are low cost, scalable, robust, adaptable, effective, flexible etc. In this paper we use a robust scheme for hand gesture recognition which uses hand detection and feature extraction using Gabor filter. This method will remove noise from the image. Swarm robots can be controlled through these gestures. The system performance and accuracy was increased with the usage of hand detection and feature extraction.

\section{References}

[1] C.C.Wang, K.C.Wang.: Hand Posture Recognition Using Adaboost with SIFT For Human Robot Interaction, in Robotics: Viable Robotic Service to Human, Springer-2009.

[2] M. Kolsch and M. Turk.: Robust hand detection, in IEEE International Conference on Automatic Face and Gesture Recognition, 2004.

[3] Cristina Manresa, Javier Varona, Ramon Mas and Francisco J.Perales: Hand Tracking and Gesture Recognition for Human-Computer Interaction, Electronic Letters on Computer Vision and Image Analysis 5(3):96-104, 2005.

[4] Alessandro Giusti, Jawad Nagi, Luca M. Gambardella, Gianni A. Di Caro: Distributed Consensus for Interaction between Humans and Mobile Robot Swarms (Demonstration).

[5] Ihab Zaqout, Roziati Zainuddin, Sapian Baba: Pixel-Based Skin Color Detection Technique, in Machine Graphics and Vision, 2005. FLEXChip Signal Processor (MC68175/D), Motorola, 1996.

[6] Qiu-yu Zhang, Mo-yi Zhang, Jian-qiang Hu,.: Hand Gesture Contour Tracking Based on Skin Color Probability and State Estimation Model, Journal of Multimedia, Vol. 4, No. 6, December 2009.

[7] Low-Cost Dual Rotating Infrared Sensor for Mobile Robot Swarm Applications Geunho Lee, Member, IEEE, and Nak Young Chong, Member, IEEE.

[8] G. Lee and N. Y. Chong, "Decentralized formation control for small scale robot teams with anonymity," Mechatronics, vol. 19, no. 1, pp.85-105, 2009.

[9] G. Lee and N. Y. Chong, “A geometric approach to deploying robot swarms,” Ann. Math. Artif. Intell. vol. 52, no. 2-4, pp. 257-280, 2009.

[10] E. Sahin, "Swarm robotics: From sources of inspiration to domains of application," in Proc. 8th Int. Conf. Simulation of Adaptive Behavior (LNCS), 2005, vol.3342, pp.10-20.

[11] H. Niwa, K. Kodaka, Y. Sakamoto, M. Otake, S. Kawaguchi, K. Fujii, Y. Kanemori, and S. Sugano, "GPS-based indoor positioning system with multi-channel pseudolite," in Proc. IEEE Int. Conf. Robot. Autom., 2008, pp. 905-910.

[12] Faraj Alhwarin, Chao Wang, Danijela Risti -Durrant, Axel Graser, Improved SIFT-Features Matching for Object Recognition, BCS International Academic Conference- Visions of Computer Science,2008.

[13] Benavidez P, Mobile robot navigation and target tracking system, System of systems engineering, $20116^{\text {th }}$ International Conference.

[14] http://en.wikipedia.org/wiki/Scale-invariant_feature_transform. 